\title{
Internalisation et recyclage du récepteur de l'insuline
}

Après avoir lié l'hormone au niveau des microvillosités de la surface cellulaire, le récepteur de l'insuline migre vers des domaines spécialisés de la membrane plasmique (invaginations mantelées ou coated pits). Ces invaginations sont le site d'internalisation (endocytose) des complexes insuline-récepteur qui s'associent ensuite avec les endosomes et les lysosomes. L'insuline est dégradée par les enzymes lysosomales alors que le récepteur peut être recyclé en direction de la membrane plasmique. Ces processus qui contrôlent le nombre de récepteurs présents à la surface cellulaire pourraient être perturbés dans certaines situations telles que le diabète et les résistances à l'insuline.

\section{Jean-Louis Carpentier}

Maître d'enseignement et de recherche à l'université de Genève

\section{ADRESSE}

J.-L. Carpentier: institut d'histologie et d'embryologie, CMU, université de Genève, 1 , rue Michel-Servet, 1211 Genève 4, Suisse.

$\mathrm{m} / \mathrm{s} n^{\circ} 2$ vol. 4, férier 88 e récepteur insulinique est une protéine intégrale de la membrane plasmique de la plupart des cellules de l'organisme. Il est constitué de deux sous-unités couplées par des ponts disulfures. Les sous-unités $\alpha$ et $\beta$ du récepteur sont synthétisées sous la forme d'une seule chaîne polypeptidique qui est ensuite clivée et à laquelle s'ajoutent des chaînes latérales glucidiques[1]. La séquence protéique du récepteur insulinique est bien établie : la sous-unité $\alpha$ est capable de lier l'insuline alors que la sous-unité $\beta$ possède une activité kinase permettant son autophosphorylation [2]. Parallèlement à sa synthèse et à son insertion dans la membrane plasmique, le récepteur insulinique peut être soustrait de la surface cellulaire par un processus appelé endocytose adsorptive. Dans cet article nous nous intéresserons plus particulièrement à ce processus d'endocytose de l'insuline et de son récep- teur et nous chercherons à en établir les implications physiologiques et physiopathologiques.

\section{Événements de surface}

La surface de la plupart des cellules envoie dans le milieu extracellulaire de fines projections appelées microvillosités. Ces microvillosités sont de longueur variable, riches en éléments du cytosquelette et dépourvues d'organelles. Ces projections procurent aux cellules une large surface de contact (figure 1, page 84).

Lorsque des cellules cibles de l'insuline telles que des adipocytes en culture (adipocytes 3T3-Ll), des hépatocytes fraîchement isolés, ou des lymphocytes humains en culture (IM-9) sont incubées en présence d'insuline couplée à de l'iode 125 ( ${ }^{125}$ I-insuline), la radioactivité peut être localisée au microscope électronique par autoradiographie quantitative [3, 4]. Si les cellules sont incubées pendant un temps court, à basse 


\begin{abstract}
RÉFÉRENCES
l. Hedo JA, Kahn CR, Hayashi M, Yamada KM, Kasuga M. Biosynthesis and glycosylation of the insulin receptor. Evidence for a single polypeptide precursor of the two major subunits. J Biol Chem 1983 ; 258 : 10020-6.

2. Kahn CR. The molecular mechanism of insulin action. Annu Rev Med 1985 ; 36 : 42951.

3. Carpentier JL, Gorden P, Amherdt M, van Obberghen E, Kahn CR, Orci L. ${ }^{125}$ I-insulin binding to cultured human lymphocytes. Initial localization and fate of hormone determined by quantitative electron microscope autoradiography. J Clin Invest 1978; 61 : 1057-70.

4. Carpentier JL, Gorden P, Freychet P, Le Cam A, Orci L. Lysosomal association of internalized ${ }^{125}$ I-insulin in isolated rat hepatocytes. Direct demonstration by quantitative electron microscopic autoradiography. J Clin Invest $1979 ; 63$ : 1249-61.
\end{abstract}

5. Carpentier JL, van Obberghen E, Gorden $\mathrm{P}$. Orci L. Surface redistribution of $125 \mathrm{I}$. insulin in cultured human lymphocytes. $J$ Cell Biol 1981 ; 91 : 17-25.

6. Orci L, Carpentier JL, Perrelet A, Anderson RGW, Goldstein JL, Brown MS. Occurence of low density lipoprotein receptors within large pits on the surface of human fibroblasts as demonstrated by freeze-etching. Exp Cell Res 1978; 113 : 1-13.

7. Heuser J, Evans L. Three dimensional visualization of coated vesicle formation in fibroblasts. J Cell Biol 1980; 84 ; 560-83.

8. Montesano R, Perrelet A, Vassali P, Orci L. Absence of filipin sterol complexes from large coated pits on the surface of cultured cells. Proc Natl Acad Sci USA 1979 ; 76 : 63915.

9. Anderson RGW, Brown MS, Goldstein L. Role of coated endocytotic vesicles in the uptake of receptor-bound low density lipoprotein in human fibroblasts. Cell $1977 ; 10$ :

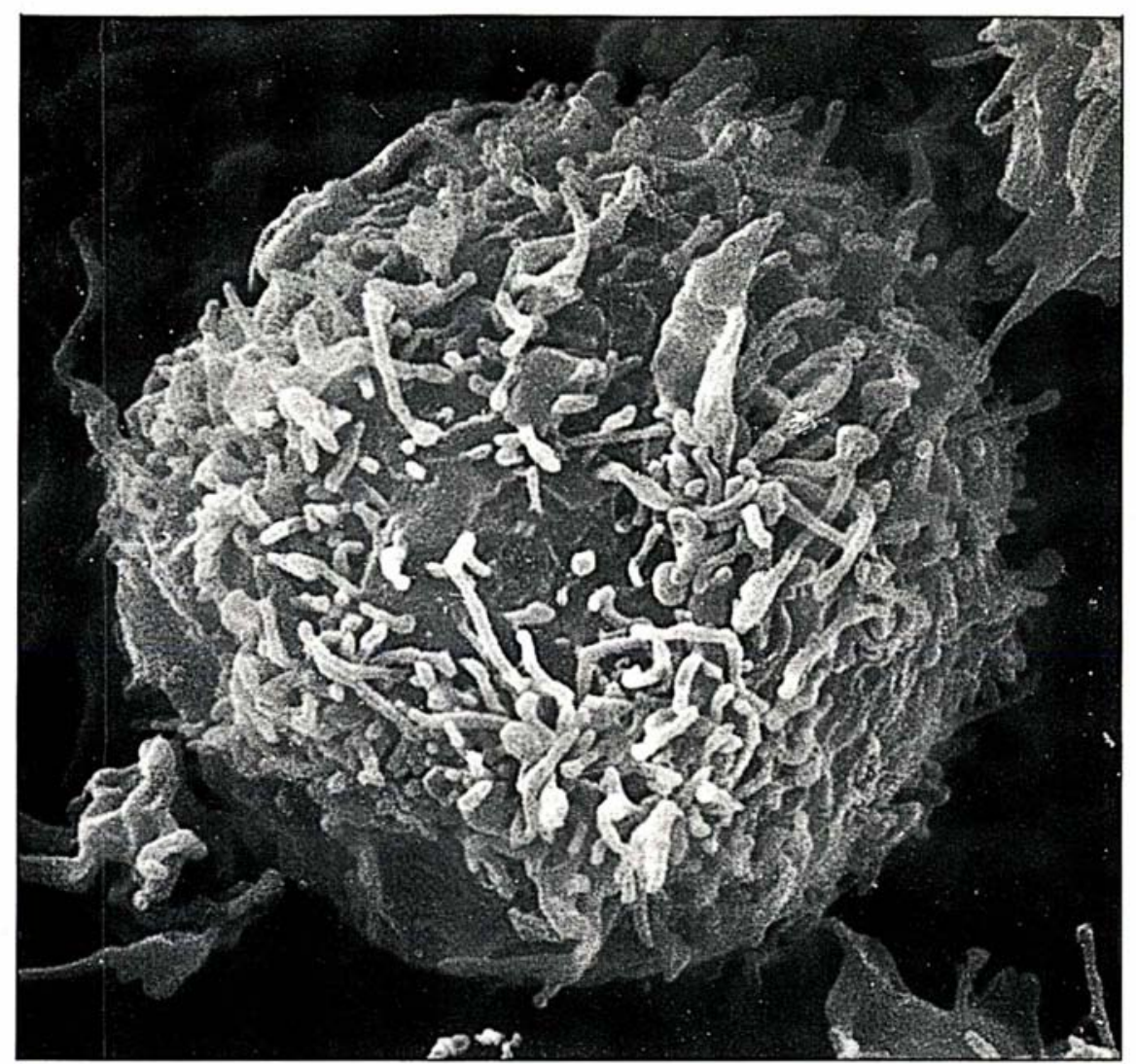

$\Delta$

température en présence du ligand radioactif (la condition la plus représentative de la liaison initiale de l'hormone aux cellules), les grains autoradiographiques sont retrouvés préférentiellement associés aux microvillosités [5]. En fonction du temps et de la température d'incubation, l'125Iinsuline se redistribue dans le plan de la membrane et se concentre progressivement dans des invaginations de surface caractérisées par la présence d'épines décorant le feuillet cytoplasmique de la membrane [5]. Ces invaginations sont des domaines spécialisés de la membrane où se concentrent de nombreux ligands tels que les lipoprotéines de faible densité (LDL) et des facteurs de croissance (figure 2). Les épines du feuillet cytoplasmique de la membrane sont constituées d'une protéine de poids moléculaire 185000 appelée clathrine ainsi que d'au moins
Figure 1. Vue générale au microscope à balayage d'un lymphocyte de la lignée IM-9. La surface de la cellule forme de nombreuses microvillosités. (d'après [5]).

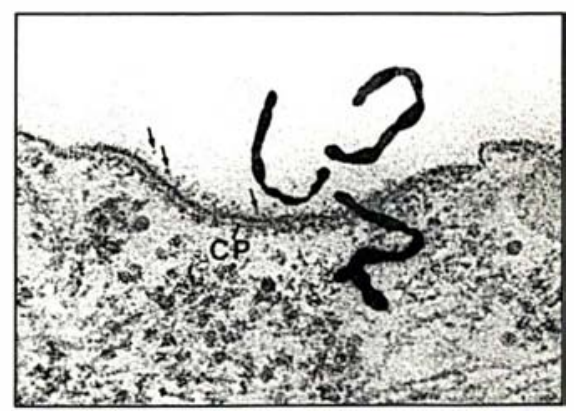

Figure 2. Coated pit (invagination mantelée) de la membrane plasmique d'un fibroblaste humain on culture, incubé en présence de LDL couplées à de la ferritine (flèches) et d'EGF marqué à l'iode 125 (grains autoradiographiques). Les deux ligands sont concentrés dans la même coated pit (CP). (d'après [27]). 
deux autres protéines de poids moléculaire inférieur. Ces invaginations, appelées coated pits (invaginations mantelées) se distinguent également par la présence d'une plus grande concentration de particules intramembranaires (correspondant aux protéines de la membrane) [6], par l'organisation tridimensionnelle de la clathrine qui forme un réseau de pentagones et d'hexagones [7] et par un manque de sensibilité à la fílipine qui forme des complexes avec le cholestérol des segments indifférenciés de la membrane [8].

\section{Endocytose}

Le rôle clé joué par les coated pits dans le processus d'endocytose adsorptive a été pour la première fois établi par Anderson et al. qui ont montré que l'internalisation des LDL s'effectuait par leur intermédiaire [9]. L'insuline, concentrée dans ces domaines, est internalisée par invagination progressive, rétrécissement du collet dont les bords fusionnent et détachement de la membrane plasmique pour former une coated vesicle (vésicule mantelée). L’absence de connexion résiduelle avec la membrane plasmique, et donc la nature vésiculaire de ces structures, a pu être vérifiée sur coupes sériées [10]. La durée de vie de ces coated vesicles est de l'ordre de 1 à 2 minutes; par un mécanisme encore mal connu, le coat (manteau) se dissocie de la vésicule, perd son organisation tridimensionnelle, et ses sous-unités (les triskélions) pourraient être réuti- lisées au niveau de la membrane plasmique pour reformer de nouveaux coats [11].

Après son association avec les

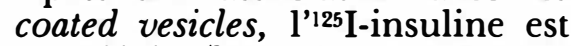
transférée dans un système de tubules et de vésicules occupant des domaines bien délimités du cytoplasme périphérique $[10,12]$ (figures 3 et 4 ). Ce système tubulovésiculaire appelé endosome a un contenu acide[13]. Cette acidification a des répercussions fonctionnelles importantes pour la majorité des complexes ligandsrécepteurs qui transitent par ce compartiment: pour certains virus, cette acidification permet à la membrane virale de fusionner avec la membrane de l'endosome et au virus d'avoir accès au cytoplasme ; dans le cas de la transferrine, l'acidification permet au

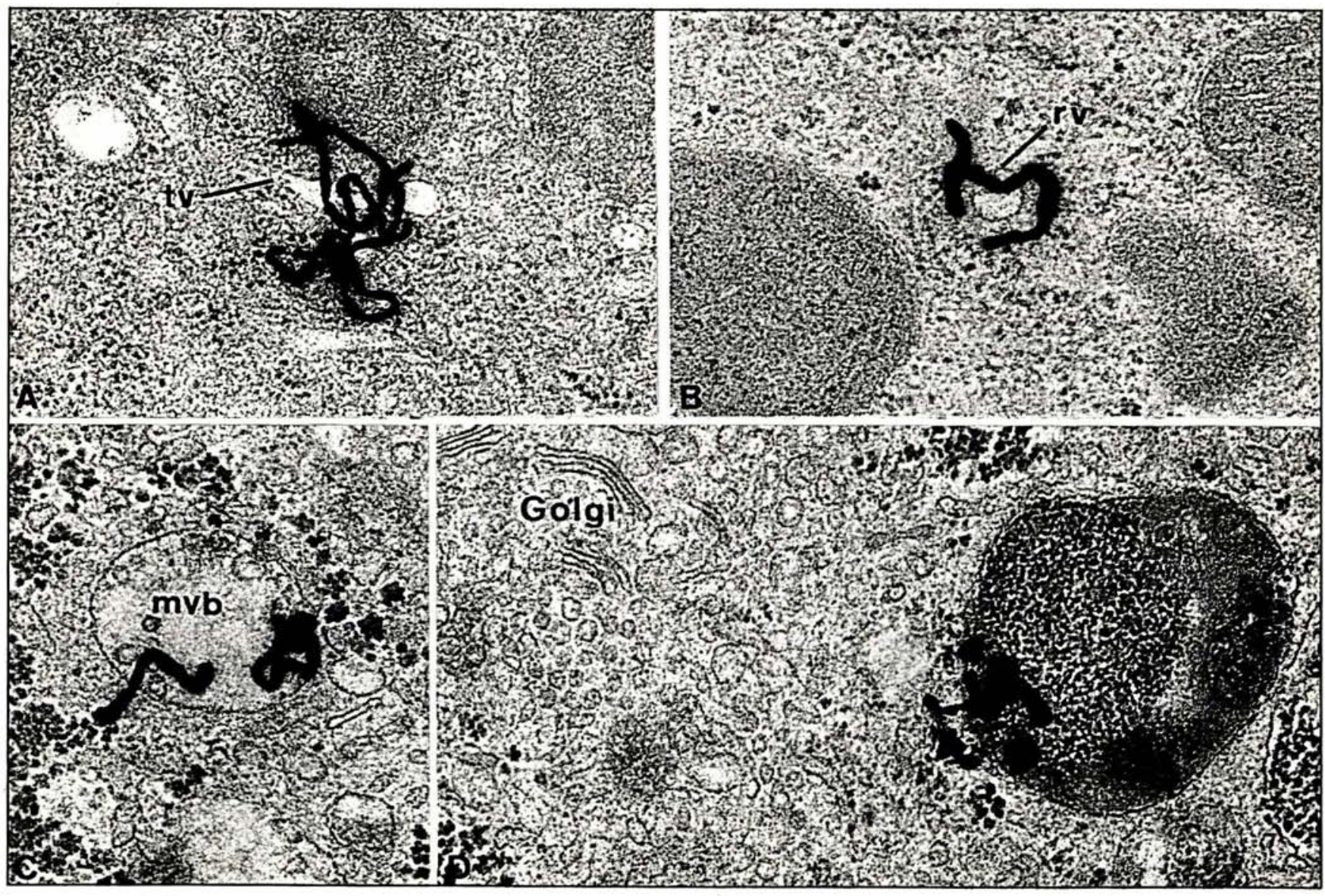

Figure 3. Coupes fines d'hépatocytes de rat incubés en présence de 125I-insuline. Les grains autoradiographiques sont associés avec: (A) le système tubulo-vésiculaire (tv) d'un endosome, (B) une vésicule de recyclage (rv), (C) un corps multivésiculaire (mvb), (D) un corps dense (lysosome) près du complexe de Golgi. 


\section{RÉFÉRENCES}

10. Fan JY, Carpentier JL, van Obberghen E, et al. Receptor-mediated endocytosis of insulin : role of microvilli, coated pits, and coated vesicles. Proc Natl Acad Sci USA 1982; 79 . 7788-91.

11. Schlossman DM, Schmid SL, Braell WA, Rothman JE. An enzyme that remove clathrin coats: Purification of an uncoating ATPase. J Cell Biol 1984 ; 99 : 723-33.

12. Tran D, Carpentier JL, Sawano F, Gorden $P$, Orci L. Ligands internalized through coated or noncoated invaginations follow a common intracellular pathway. Proc Natl Acad Sci USA 1987; 84 : 7957-61.

13. Helenius A, Mellman I, Wall D, Hubbard A. Endosomes. Trends Biochem Sci 1983 ; 8 : 245-50.

14. Carpentier JL, Gorden P, Robert A, Orci L. Internalization of polypeptide hormones and receptor recycling. Experientia $1986 ; 42$ : 734-44.

15. Fehlmann M, Carpentier JL, Le Cam A et al. Biochemical and morphological evidence that the insulin receptor is internalized with insulin in hepatocytes. J Cell Biol 1982; 93 : 82-7.

16. Carpentier JL, Gazzano H, van Obberghen $E$, Fehlmann $M$, Freychet $P$, Orci L. Intracellular pathway followed by the insulin receptor covalently coupled to ${ }^{125} \mathrm{I}$-photoreactive insulin during internalization and recycling. J Cell Biol 1986; 102 : 989-96.

17. Brown MS, Anderson RGW, Goldstein JL. Recycling receptors : the round-trip itinerary of migrant proteins. Cell $1983 ; 32$ : 6637.

18. Carpentier JL. Dayer JM, Lang U, Silverman R, Orci L, Gorden P. Down regulation and recycling of insulin receptors : effect of monensin on IM-9 lymphocytes and U-937 monocyte-type cells. J Biol Chem 1984 ; 259 : 14190-5.

19. Davies PJA, Davies DR, Levitski A et al. Transglutaminase is essential in receptormediated endocytosis of $\alpha_{2}$-macroglobulin and polypeptide hormones. Nature 1980 ; fer de se dissocier de son transporteur et d'avoir accès au cytoplasme; pour de nombreux autres ligands, l'acidification induit une dissociation du ligand de son récepteur, ce qui amène chacun d'eux à être séquestré dans des domaines distincts et de suivre des processus différents [12].

L'étape suivante du parcours intracellulaire de l'insuline est son association à des corps multivésiculaires $[4,10,13,14]$ (figures 3 et 4). La biogenèse de ces structures reste mystérieuse: sont-elles formées de novo ou d'éléments endosomiaux? Cela reste à établir. Quoiqu'il en soit, les corps multivésiculaires semblent être des structures intermédiaires dans un processus continu, la structure suivante étant le lysosome caractérisé par son contenu riche en enzymes protéolytiques actives à $\mathrm{pH}$ acide. La distinction entre corps multivésiculaires et lysosomes est relativement floue; aussi, pour plus de facilité, nous incluerons ces deux types de structures sous la terminologie « lysosome » dans la suite de cette revue.

\section{Recyclage des récepteurs}

Grâce à des études morphologiques et biochimiques impliquant l'utilisation de dérivés photoréactifs capables de se lier de façon irréversible aux récepteurs insuliniques, il a été possible de montrer que ce récepteur était internalisé en même temps que l'hormone [15]. Ces mêmes expé-

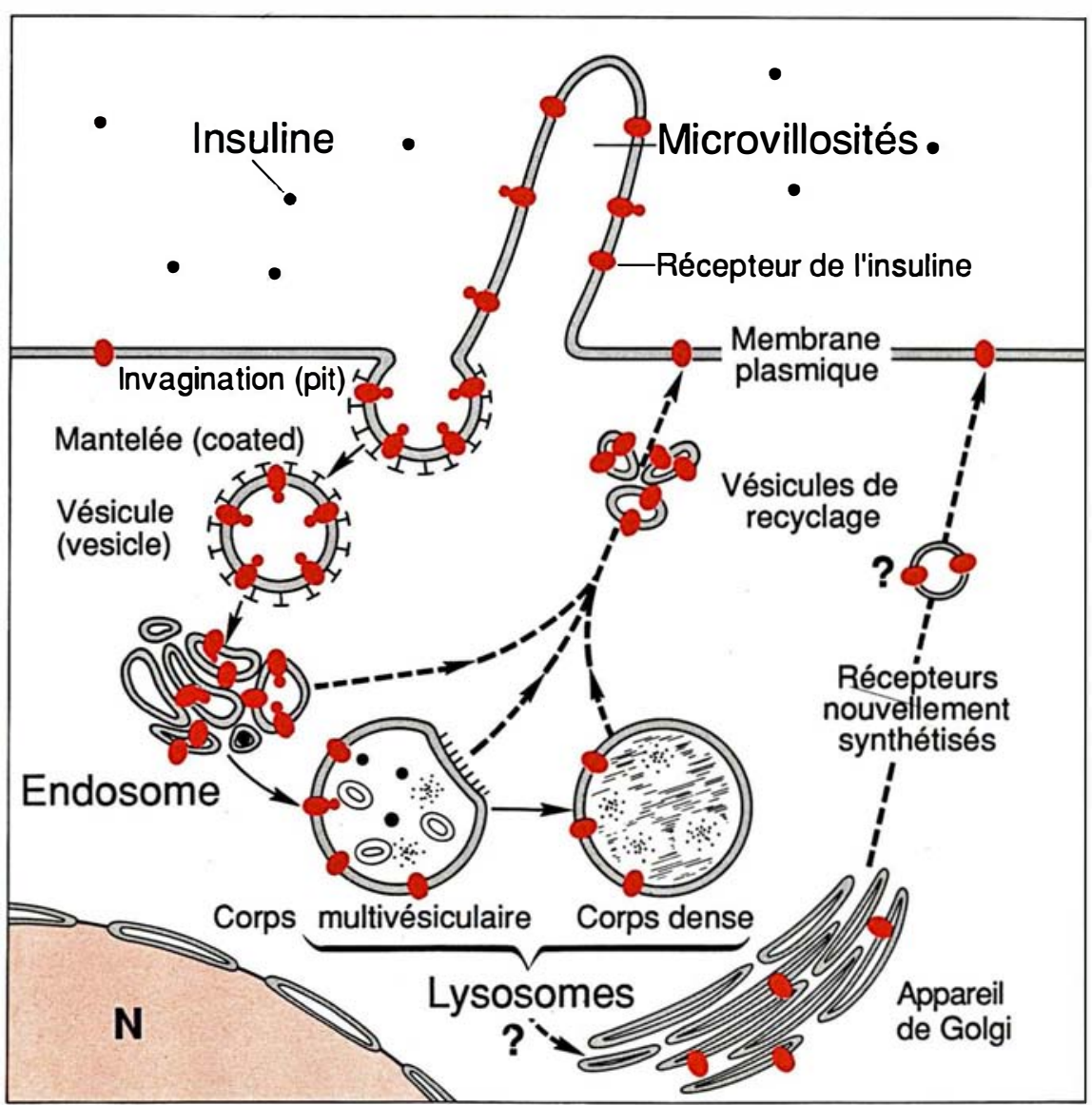

Figure 4. Schéma représentatif du trajet suivi par l'insuline et son récepteur spécifique à la suite de leur interaction initiale à la surface d'une cellule cible. Les membranes sont représentées en gris; les récepteurs sont en rouge; $N=$ noyau. 
riences ont également démontré que, après son internalisation, le récepteur insulinique était recyclé en direction de la membrane plasmique [16]. La séparation du ligand et du récepteur n'est pas requise pour permettre le recyclage. En effet, l'insuline photoréactive couplée de façon covalente au récepteur est recyclée en même temps que lui. Ce recyclage, qui s'effectue via les lysosomes, est cependant très lent ; il est donc probable qu'une autre voie plus rapide nécessite une dissociation du ligand et, comme cela est démontré pour nombre de récepteurs, survient à une étape précoce de l'internalisation, vraisemblablement au niveau des endosomes [13] (figure 4).

\section{Induction et contrôle de l'endocytose}

L'endocytose adsorptive est un système d'internalisation très efficace puisque le ligand est concentré aux sites d'endocytose (coated pits). Il est cependant important de noter que la localisation initiale des récepteurs inoccupés peut être différente d'un récepteur à l'autre. Certains récepteurs, tels les récepteurs aux protéines de transport (récepteurs des LDL, récepteurs de la transferrine, etc.), sont associés aux coated pits même dans leur forme inoccupée; ces récepteurs occupés ou non sont donc internalisés et recyclés de façon continue [17]. Ils fonctionnent comme des tapis roulants qui tournent continuellement, indépendamment de la présence de passagers (les ligands). En revanche, les récepteurs insuliniques, comme la plupart des récepteurs pour les hormones polypeptidiques et les facteurs de croissance, sont, dans leur forme inoccupée, en dehors des coated pits (tel le récepteur insulinique associé aux microvillosités). De tels récepteurs ont besoin de la liaison à leur ligand spécifique pour être transportés vers les coated pits. Ils fonctionnent comme des ascenseurs qui ne se mettent en mouvement que si $m / s n^{\circ} 2$ vol. 4, févier 88

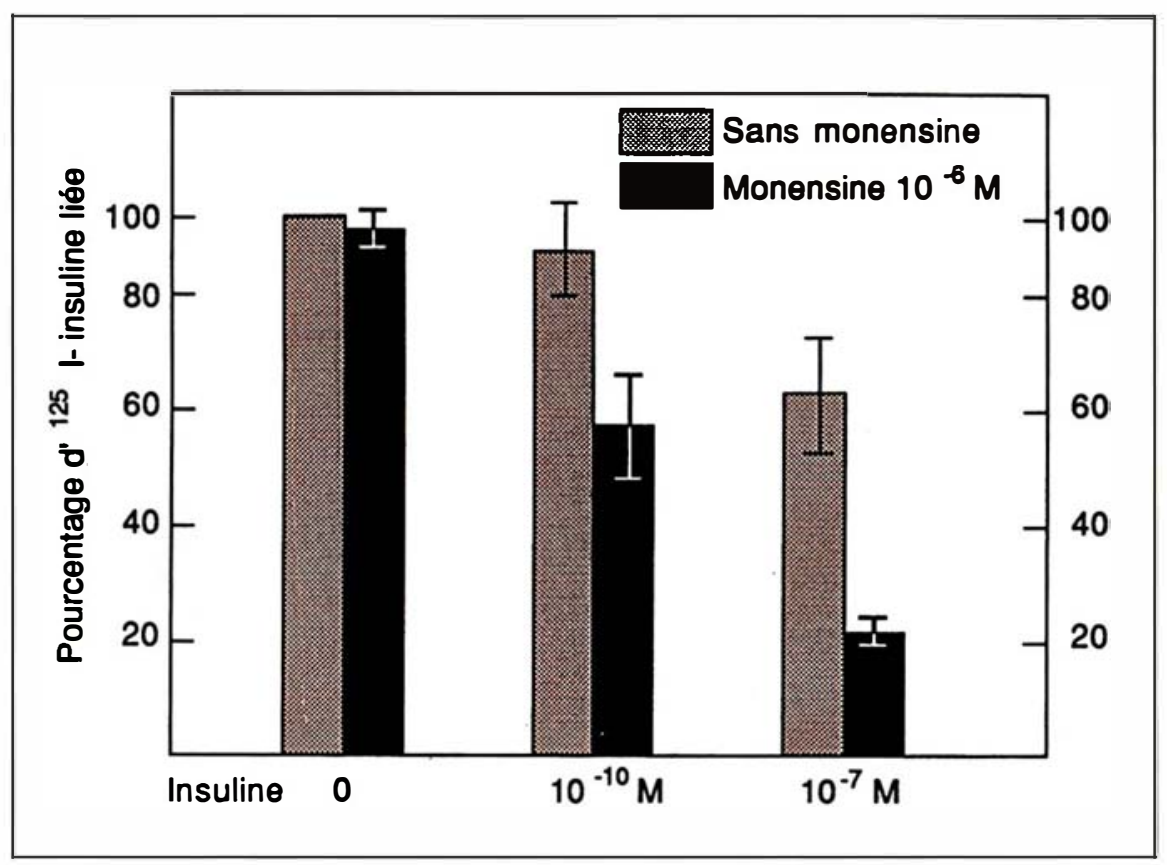

Figure 5. Effet d'un inhibiteur du recyclage sur la capacité des cellules U-937 à lier la 125/-insuline. La monensine n'a pas d'influence en l'absence d'insuline mais, en revanche, elle accroît la perte de récepteurs insuliniques de surface induite par l'insuline.

un passager en fait la demande. Dans un tel contexte, il n'est donc pas étonnant de noter qu'un inhibiteur du recyclage des récepteurs (la monensine) induit une perte de récepteur LDL de surface même en l'absence de ligand, alors que la même drogue n'a d'effet sur le nombre de récepteurs insuliniques de surface qu'en présence d'insuline [18] (figure 5).

Le mécanisme par lequel l'insuline induit l'internalisation de son propre récepteur est mal connu. Il a été suggéré que les transglutaminases pourraient être impliquées dans ce processus d'induction. Cette hypothèse avait comme point de départ l'observation d'un parallélisme entre l'inhibition de l'internalisation et l'inhibition de l'activité transglutaminasique produites par une série d'agents chimiques [19]. Il est maintenant évident que d'autres agents inhibiteurs des transglutaminases n'ont pas d'effet sur l'endocytose, excluant l'hypothèse de départ [20]. Plus récemment, il a été démontré que les récepteurs pour divers hormones polypeptidiques et facteurs de croissance (EGF, PDGF, insuline) s'autophosphorylaient à la suite de la liaison de l'hormone ou du facteur de croissance [2]. On a dès lors suggéré que cette phosphorylation pourrait représenter le mécanisme d'induction de l'internalisation. Des études récentes montrant qu'un récepteur insulinique ne disposant pas d'activité tyrosine kinase n'est pas internalisé sont en accord avec ce concept [21]. En revanche, nous avons montré qu'un anticorps anti-récepteur insulinique est internalisé à la même vitesse que l'insuline en dépit du fait qu'il n'induit pas d'autophosphorylation du récepteur insulinique [22]. En conséquence, la phosphorylation du récepteur insulinique ne semble pas être le mécanisme général d'induction de l'internalisation du récepteur insulinique. En ce qui concerne le contrôle du processus d'internalisation, nous avons récemment testé le rôle de deux candidats potentiels: la concentration calcique intracellulaire et la protéine kinase C. S'il 


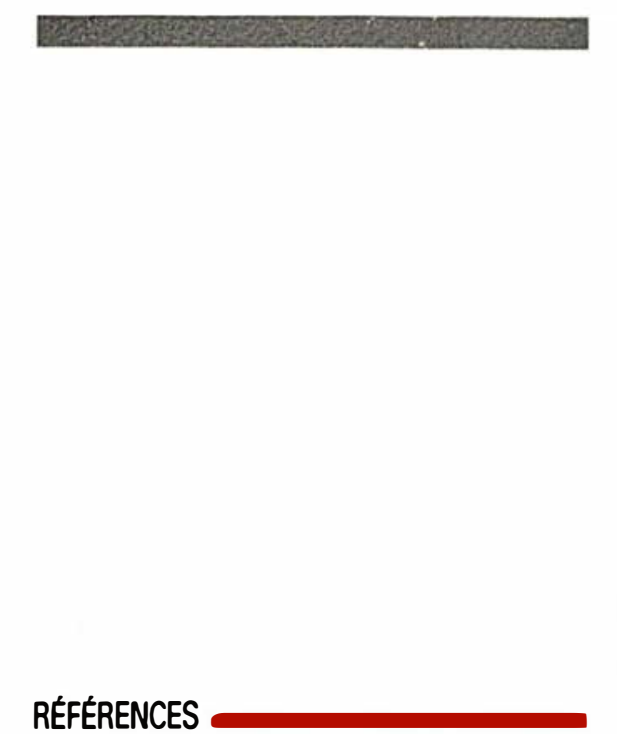

20. Haigler HT, Willingham MC, Pastan I. Inhibitors of 125 I-epidermal growth factor internalization. Biochem Biophys Res Commun 1980 ; 94 : 630-7.

21. McClain DA, Maegawa H, Lee J, Dull $\mathrm{TJ}$, Ulrich A, Olefsky JM. A mutant insulin receptor with defective tyrosine kinase displays no biologic activity and does not undergo endocytosis. J Biol Chem 1987 ; 262 : 14663-71.

22. Kasuga M, Carpentier JL, van Obberghen E, Orci L, Gorden P. ${ }^{125}$ I-anti-insulin receptor Fab is internalized by cultured human lymphocytes. Biochem Biophys Res Commun 1983; 114 : 230-3.

23. Iacopetta B, Carpentier JL, Pozzan T, Lew DP, Gorden P, Orci L Role of intracellular calcium and protein kinase $C$ in the endocytosis of transferrin and insulin by HL60 cells. J Cell Biol 1986; 103 : 851-6.

24. Goldstein JL, Brown MS, Anderson RGW, Russel DN, Schneider WJ. Receptormediated endocytosis: concepts emerging from the LDL receptor system. Ann Rev Cell Biol 1985 ; 1 : 1-39.

25. Gorden P, Freychet P, Carpentier JL, Canivet B, Orci L. Receptor-linked degradation of ${ }^{125}$ I-insulin is mediated by internaliza tion in isolated rat hepatocytes. J Biol Med $1982 ; 55: 101-12$

26. Carpentier JL, Robert A, Grunberger G et al. Receptor-mediated endocytosis of polypeptide hormones is a regulated process: inhibition of ${ }^{125}$ I iodoinsulin internalization in hypoinsulinemic diabetes of rat and man. J Clin Endoctrinol Metab 1986; 63 : 151-5.

27. Carpentier JL, Gorden P, Anderson RGW, et al. Co-localization of 125 I-epidermal growth factor and ferritin-low density lipoprotein in coated pits : a quantitative electron microscopic study in normal and mutant

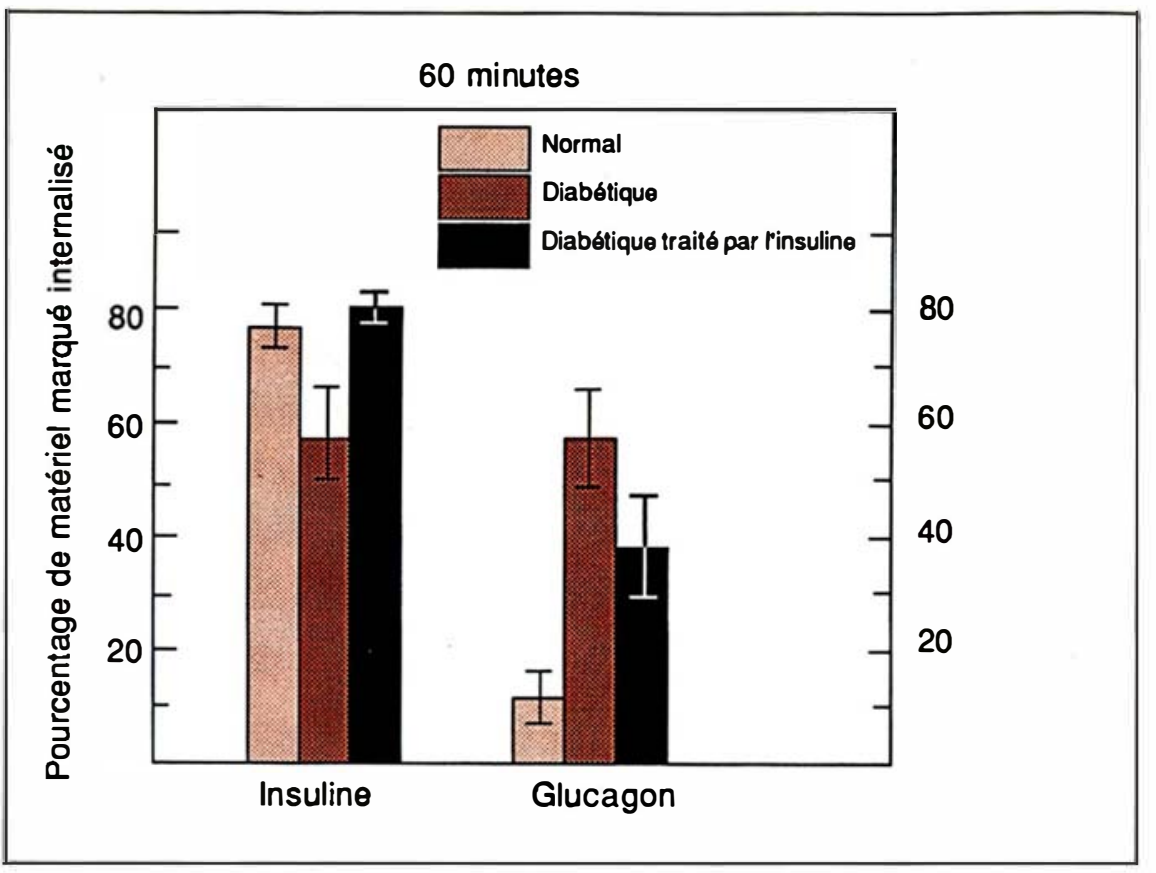

Figure 6. Internalisation de 125/-insuline et de 125I-glucagon dans des hépatocytes de rats contrôles (rectangles roses), de rats rendus diabétiques à l'aide de streptozotocine (rectangles tramés rouges) et de rats diabétiques traités à l'insuline (rectangles noirs).

s'avère que des variations de la concentration calcique intracellulaire n'influencent pas l'internalisation de l'insuline, une stimulation de la protéine kinase $\mathrm{C}$ par un ester du phorbol induit en revanche une augmentation significative de l'internalisation de ce ligand [23]. Des changements de l'activité de la protéine kinase $C$ (par exemple, à la suite de la formation d'un complexe ligand-récepteur capable d'induire une production de diacylglycérol, l'activateur physiologique de la protéine kinase $\mathrm{C}$ ) pourraient donc moduler l'internalisation des récepteurs insuliniques. De la sorte, les cellules pourraient disposer d'un mécanisme de communication entre des récepteurs pour différents ligands.

\section{Implications physiologiques}

Les implications physiologiques de l'endocytose adsorptive dépendent essentiellement du ligand considéré. Dans le cas des récep- teurs des LDL, l'internalisation des lipoprotéines contenant le cholestérol permet une libération du cholestérol grâce à l'intervention des enzymes lysosomales. Ce cholestérol libre module alors l'activité d'enzymes intracellulaires et des récepteurs aux LDL eux-mêmes [24]. Dans le cas des hormones polypeptidiques et des facteurs de croissance, le rôle direct de l'internalisation du complexe hormone-récepteur dans la transmission du signal biologique reste obscur. Ce processus paraît en revanche impliqué dans la dégradation de l'hormone et le contrôle de la concentration de récepteurs de surface. Ainsi que nous l'avons rapporté auparavant, l'insuline s'associe avec les lysosomes qui sont des organelles intracellulaires impliqués dans la dégradation de matériel intra- et extracellulaire. La démonstration d'une réduction de la dégradation de l'insuline en présence de substances capables d'alcaliniser les lysosomes et donc de réduire leurs capacités protéolytiques a permis 
de démontrer le rôle de ces structures, et donc du processus d'internalisation, dans la dégradation de l'insuline [25]. Une telle observation n'exclut cependant pas la possibilité d'une dégradation par des protéases neutres dont le site d'action pourrait être localisé au niveau de la membrane plasmique.

D'autre part, l'insuline et le récepteur insulinique étant internalisés simultanément, le processus d'internalisation permet aux cellules de moduler la concentration de récepteurs insuliniques de surface [14]. Cependant, il n'existe pas de corrélation systématique entre l'internalisation de l'insuline et la perte de récepteurs de surface. Ceci est dû au fait que le contrôle du nombre de récepteurs de surface dépend non seulement de l'internalisation mais également du recyclage, de la dégradation et de la synthèse des récepteurs. Dans les cellules U-937, de faibles concentrations d'insuline induisent une perte de récepteurs insuliniques de surface. Ces récepteurs sont, en partie, retrouvés à l'intérieur des cellules. Il est donc clair que, dans ce cas, les récepteurs sont transférés à l'intérieur de la cellule où ils sont soit dégradés soit préservés dans certains compartiments intracellulaires avant d'être recyclés. La monensine, qui inhibe le recyclage, augmente la perte de récepteurs insuliniques de surface (figure 5). Comme cette drogue n'influence pas la fraction de récepteurs destinés à être dégradés, ces observations démontrent donc l'implication du recyclage des récepteurs dans la régulation du nombre des récepteurs présents à la surface de la cellule.

\section{Implications physiopathologiques}

Le diabète insulino-dépendant (type l) est caractérisé par une hypo-insulinémie et une augmentation du nombre de récepteurs insuliniques de surface. S'il est vrai que l'endocytose est un mécanisme qui permet de moduler le nombre de récepteurs in$\mathrm{m} / \mathrm{s} n^{\circ} 2$ vol. 4, févier 88 suliniques de surface, on peut s'attendre à des altérations de ce processus dans une telle situation pathologique. En effet, dans l'hypo-insulinémie, induite chez le rat par la streptozotocine, l'internalisation de l'insuline est réduite. Ce défaut d'internalisation est corrigé par un traitement insulinique et est spécifique de l'insuline puisque l'internalisation du glucagon est augmentée dans cette situation pathologique caractérisée par une hyperglucagonémie (figure 6). Une même réduction de l'internalisation de l'insuline a été observée dans des monocytes fraîchement isolés de patients diabétiques insulinodépendants [26].

Il apparaît donc que, dans des états d'hypo-insulinémie, les cellules essayent de s'adapter à cette situation pathologique en augmentant le nombre de récepteurs insuliniques de surface par une réduction du processus d'internalisation.

\section{Conclusion}

Après son association à un récepteur spécifique présent à la surface de la cellule, l'insuline, couplée à ce récepteur, pénètre à l'intérieur de la cellule et le complexe insuline-récepteur est entraîné dans une suite d'événements qui ont pour conséquence de contrôler la concentration d'insuline et de récepteurs à la surfacede la cellule. Une séquence d'événements similaires a été observée pour d'autres hormones polypeptidiques, pour des facteurs de croissance et, ce qui pourrait paraître plus surprenant, pour des ligands aussi différents que les LDL, des toxines, des virus ou des lectines. Une meilleure connaissance de la biologie cellulaire du récepteur pour les LDL a permis de mieux comprendre le métabolisme du cholestérol. On peut donc espérer que, de la même manière, l'élucidation des particularités de la biologie cellulaire du récepteur de l'insuline conduira à une meilleure compréhension de certains aspects de la pathogénie du diabète et de la résistance à l'insuline

\section{Summary}

Following insulin binding on microvilli at the cell surface, the insulin receptor migrates in the plane of the membrane and concentrates in coated pits. These invaginations prick off to form vesicles inside the cytoplasm and the insulin receptor complexes sequentially associate with endosomes and lysosomes. The insulin is degraded by lysosomal enzymes while the insulin receptor may be recycled back to the cell surface. This internalization recycling process controls the number of surface insulin receptors. It could be modified in pathological conditions such as diabetes and insulin resistance states.

\section{TIRÉS A PART}

J.-L. Carpentier: institut d'histologie et d'embryologie, $\mathrm{CMU}$, université de Genève, 1 , rue Michel-Servet, 1211 Genève 4, Suisse. 